\title{
A new satellite repeater amplifier characterization system for large bandwith NPR and modulated signals measurements
}

\author{
Alain Mallet, Françis Gizard, T. Reveyrand*, Luc Lapierre, Jacques Sombrin \\ CNES - Toulouse Space Center - 18, av. Edouard Belin, 31401 Toulouse cedex 4, France \\ (*) IRCOM - 123, av. Albert Thomas, 87060 Limoges cedex, France \\ alain.mallet@cnes.fr-francis.gizard@cnes.fr
}

\begin{abstract}
Power consumption and dissipation of satellite payloads for space telecommunication systems are mainly due to power amplifiers. To increase system capacities with limited bandwidth, multicarrier operations are required. Linearity is specified to limit the signal distorsion by the nonlinear power amplifiers. In order to minimize power consumption under those linearity requirements, accurate measurements have to be performed. The NPR is well known as the figure of merit for the intermodulation distortion performance of amplifiers in telecommunications. In this paper, the development of an accurate large bandwidth NPR measurement system is presented. It enables characterizations up to $\mathrm{Ka}$ band $(17-21 \mathrm{GHz}$ and $27-31 \mathrm{GHz}$ for space telecommunications applications with typically $250 \mathrm{MHz}$ bandwidth). Measurements made on power amplifiers have been used to optimize operating conditions.
\end{abstract}

\section{INTRODUCTION}

To answer to the considerable increase of very high capacity need, and according to the limited frequency bandwidth available, satellite communications systems often require multicarrier operations mode. The distortions due to the power amplifiers have to be controlled, thus lead to back-off of the output power. This results in an increase of dissipated and consumed power. In order to optimize the trade-off between linearity and DC power consumption, a new criterion has been proposed [1]-[2]. This criterion can take into account all types of perturbation (noises, intermodulations, inter channel interferences and external channels interferences). It gives an objective parameters to compare different technologies and to optimize the nominal power and the operating point of the chosen device. Very accurate NPR measurements are needed to lower the margin taken on consumption power.

The first goal of the measurement system presented hereafter is to perform those accurate large bandwidth (typically $250 \mathrm{MHz}$ today) NPR measurements up to Ka band (especially : $12,5-14,5 \mathrm{GHz}, 17-21 \mathrm{GHz}$ and $27-31 \mathrm{GHz}$ for space telecommunications applications).
Secondly, it's a very attractive experimental tool to optimize operating conditions of power amplifier or any subsystem or system.

And thirdly, the opportunity to characterize a component, subsystem or system degradations on modulated complex signals has been taken into account.

After the detailed description of the test-bench, different applications will be evaluated.

\section{DESCRIPTION OF THE PROPOSED CHARACTERISATION SYSTEM}

This work started with CNES R\&D activity entrusted to IRCOM. It consisted of the development of NPR measurement system in L/S band based on digital signal generation [3]. The aim of the proposed system is to extend this technique up to Ka-band with higher bandwidths.

\section{A. Overall structure}

To fulfill these objectives, especially in terms of bandwidth, frequency ranges and evolutions, the measurement system is based on :

- numerical generation of signals based on Arbitrary Waveform Generator (AWG 520 - Tektronix). A wide variety of base-band signals can be easily generated. Its main characteristics are the sample frequency $(1 \mathrm{Gech} / \mathrm{s})$ and the vertical resolution $(10$ bits).

The key advantages of digital techniques [4] over analog ones [5] are now well-known for NPR determination [6] : the shape of the noise is not imposed by a filter, it exhibits better repeatability ; and as the generated signal is pseudo-noise, the averaging of signals along several periods reduces the thermal noise.

Moreover, the numerical technique has been specially selected according to the wide variety of signals which can be generated thanks to the high degree of flexibility of the system. 
- A frequency plan with two levels of frequency translation. The determination of the intermediate frequency $(3.875 \mathrm{GHz})$ results from trade-off between modulator/demodulator performances and the facility to reject spurious frequencies after RF frequency translation. The generic part delivers signals around $3,875 \mathrm{GHz}$. This frequency plan enables a very wide range of frequencies for the characterization. In each case, specific elements are the power driver and the filter. For example, the overall architecture is given Fig. 1 for $17-21 \mathrm{GHz}$ measurements.

- To analyze the output waveform and calculate NPR, a digital oscilloscope solution has been chosen. Now, we use FFT on captured waveform to calculate NPR.

- In some cases (CDMA communication systems for example) the NPR is not well-suited to determine the nonlinear distortion due to the Device Under Test (D.U.T.) [7]-[8]. After specific calibration procedure, the signal waveforms at the D.U.T. ports will be extracted. Then, the accurate determination of the distortion due to the D.U.T. on those signals will be possible. This work is under development.

\section{B. Numerical signal calculation}

The pseudo-noise signal is created by combining a large number of discrete spectral lines equally spaced with the same amplitude and random phases. The number of the spectral lines and their phases are the signal key parameters. The notch width determination comes from a trade-off. Too wide, the signal properties are significantly changed ; too tight, the low number of tones inside the notch is not sufficient to determine accurately the noise power.

A good configuration is obtained with Matlab software considering 25000 signal tones and 5\% relative notch width. The right and left side bands have to be uncorrelated in order to excite phase and amplitude noises. If not [9], the RF signal is amplitude modulated only and does not represent multicarrier loading. It gives pessimistic results compared to NPR. Amplitude modulation is not used in satellite communications system.
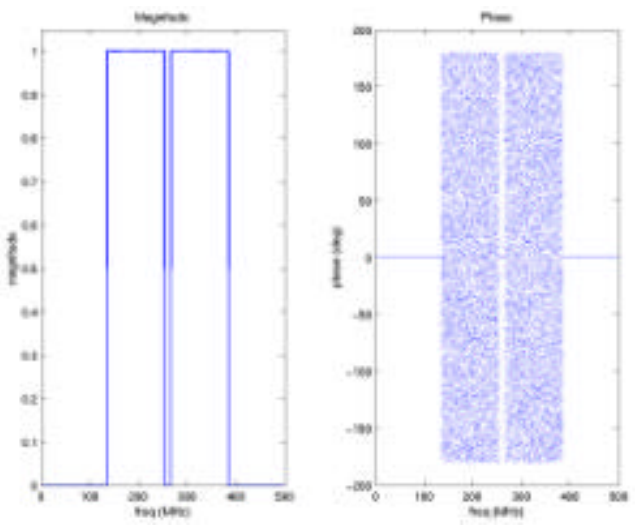

Fig. 2. Magnitude and Phase of the signal spectrum

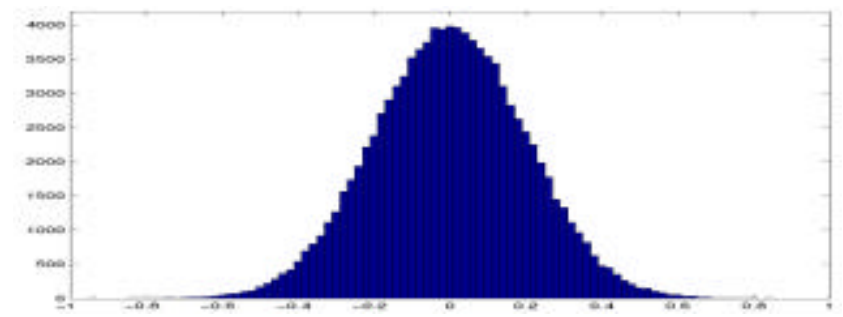

Fig. 3. Temporal signal magnitude distribution

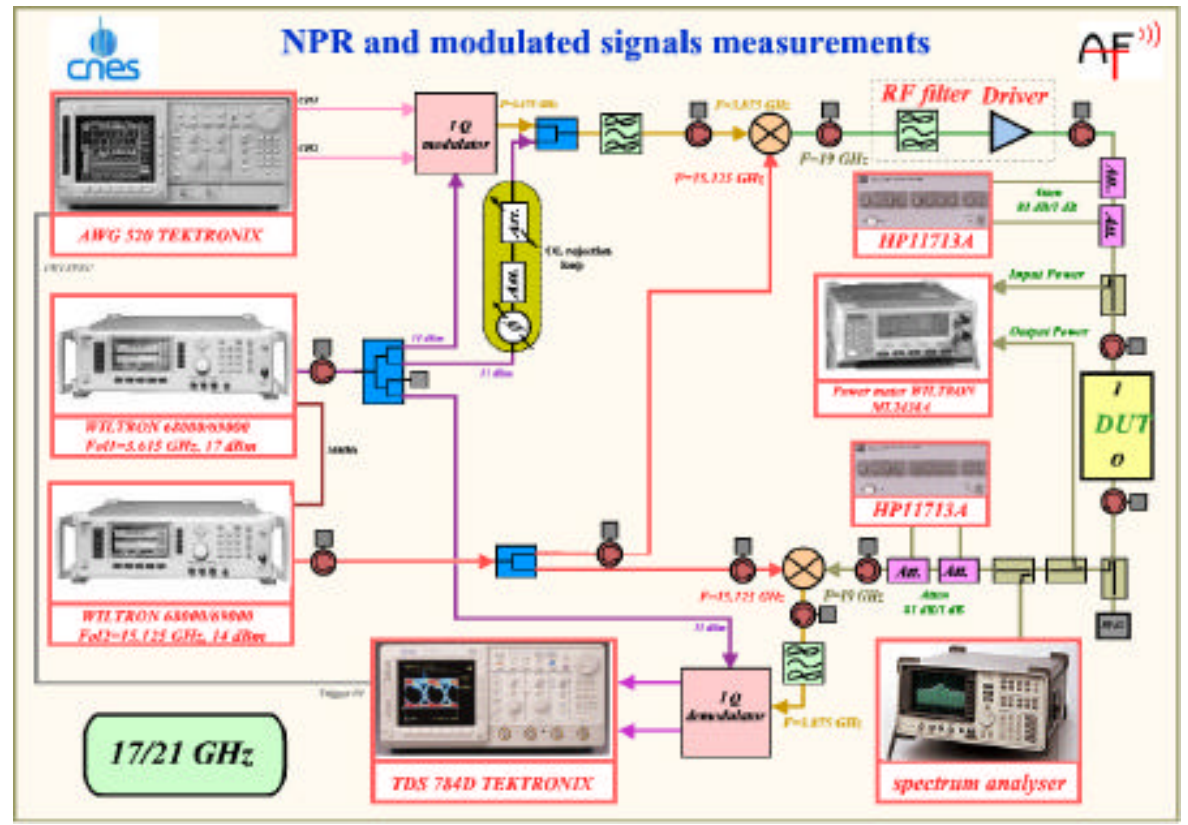

Fig. 1. Architecture in $17-21 \mathrm{GHz}$ configuration 


\section{Implementation}

The picture hereafter presents an overview of the system.

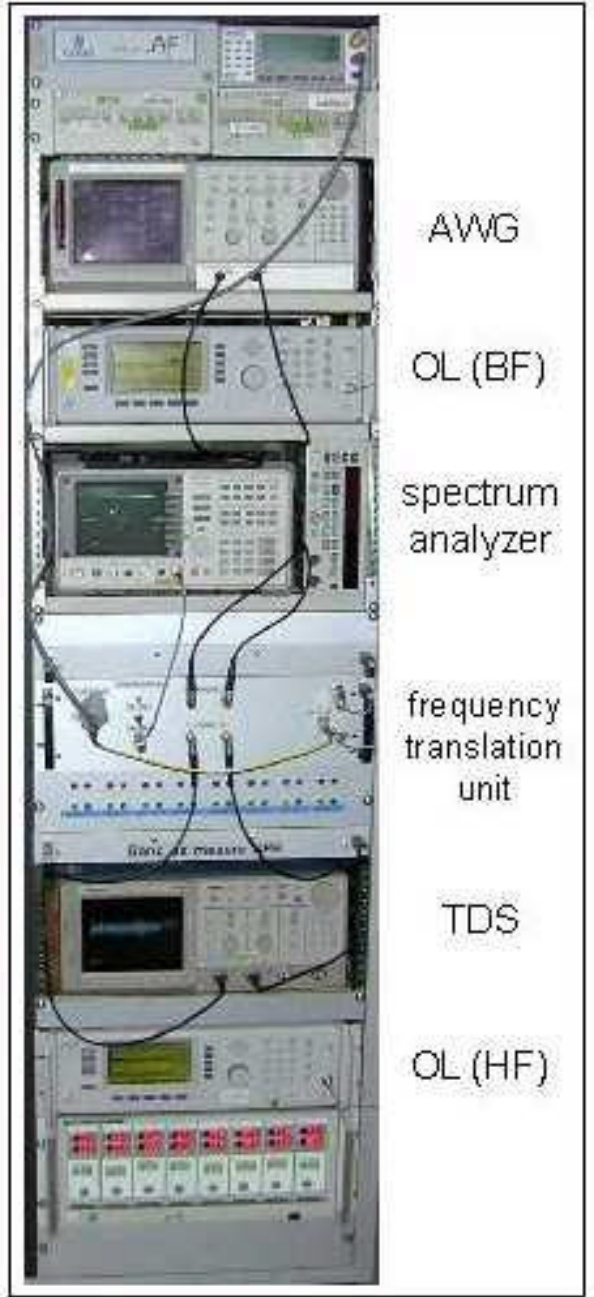

Fig. 4. Implementation of the measurement system

\section{NPR measurements system performances}

- the shape of the signal at the D.U.T. input

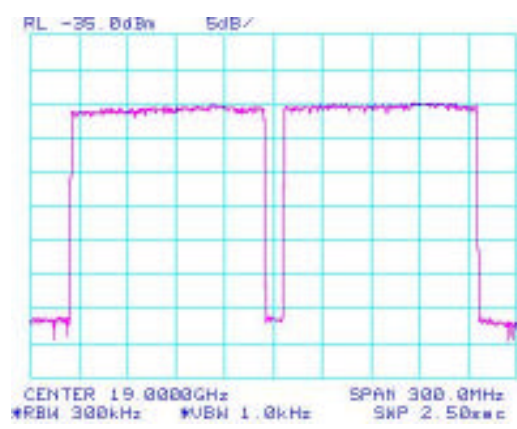

Fig. 5. $19 \mathrm{GHz} / 250 \mathrm{MHz}$ signal shape
The shape observed demonstrates a very flat profile and a notch with steeply sides.

\section{- $\quad$ validation of the signal calculation}

In order to determine the variance of the NPR due to the limited number of tones and the associated phases distribution, ten different signals are generated and applied to the D.U.T. (1W/17-21GHz SSPA from ADVANCED MICROWAVE)

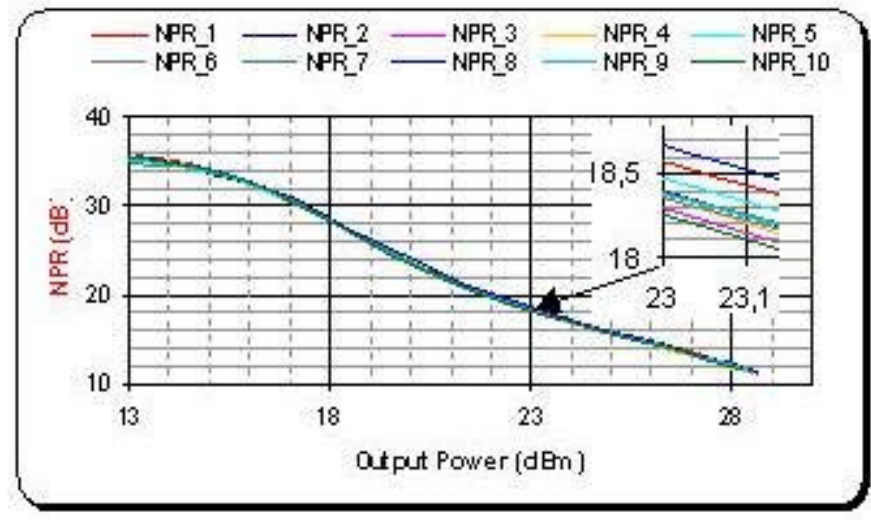

Fig. 6. $19 \mathrm{GHz} / 250 \mathrm{MHz}$ signal shape

We can note the $35 \mathrm{~dB}$ NPR at low output power level that ensures high dynamic NPR measurement. A low dispersion of $0,4 \mathrm{~dB}$ is typically obtained.

\section{- level of reproducibility}

Different measurements of the same device have been performed on a 15 days period. The figure below illustrates the high level of NPR measurements reproducibility (maximum dispersion of $0,4 \mathrm{~dB}$ ).

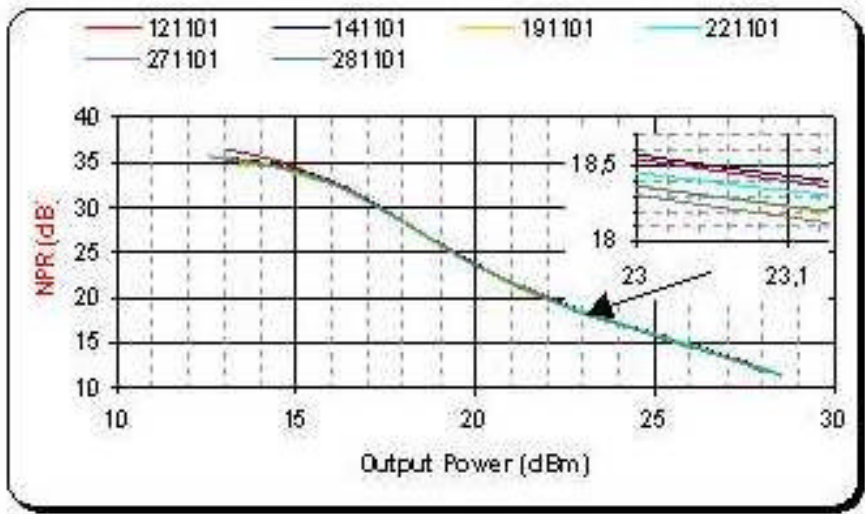

Fig. 7. NPR measurements reproducibility illustration

\section{APPLICATIONS}

The three main applications of the test-bench are now presented. 


\section{A. Accurate measurement of wide bandwidth NPR}

NPR measurements have been performed on TWTA operating at $19 \mathrm{GHz}$ under $250 \mathrm{MHz}$ pseudo-noise signal.

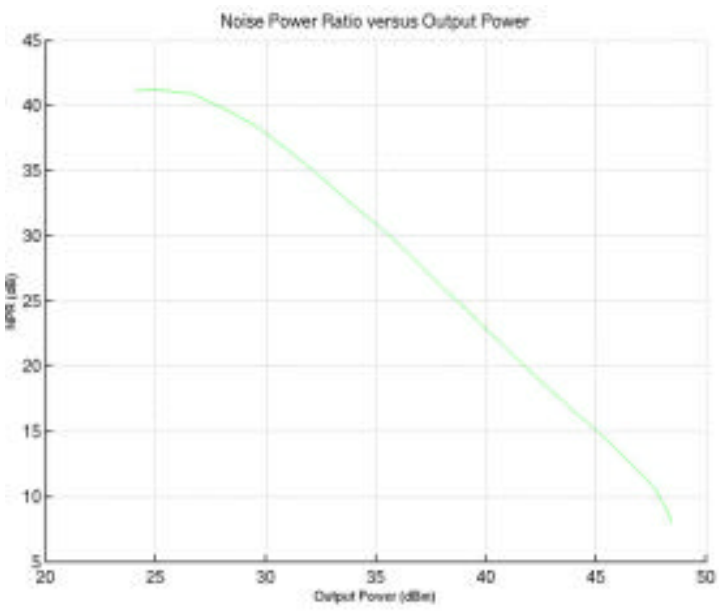

Fig. 8. NPR versus output power

B. Experimental optimization of D.U.T. operating conditions

The evaluation of the influence of helix voltage on TWTA (courtesy of Thomson Electron Devices) NPR under multicarrier output power constraint is presented hereafter.

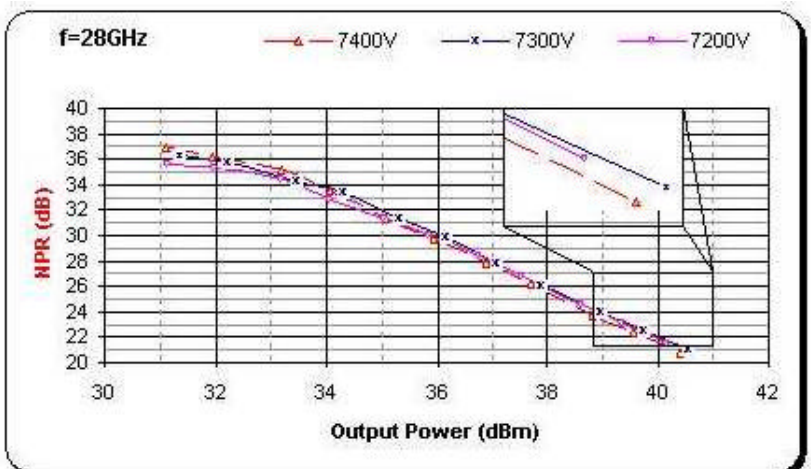

Fig. 9. NPR versus output power for three different helix voltages

The $7300 \mathrm{~V}$ helix voltage exhibits the higher NPR for the $40 \mathrm{dBm}$ required power.

\section{Modulated signal generation and acquisition}

The possibility we have to generate and acquire signals very similar to applications ones is very attractive. This work is under development and first results will be available soon.

\section{CONCLUSION}

A digital generation/acquisition and large bandwidth NPR test-bench have been developed for optimization of telecommunication satellite repeaters up to Ka band. High dynamic and very high level of reproducibility have been demonstrated. Very accurate NPR measurements enable to determine precisely nominal power and operating point of power amplifiers. This leads to decrease the power consumption of satellite payloads.

The measurement system has the capability to generate and acquire a wide variety of modulated signal. It enable to determine the distortion of the nonlinear amplifier in some cases where the NPR is not well-suited. Furthermore, this concept allows to generate exactly the same signals as software simulators. In particular, it can be used to extract and validate behavior models for nonlinear memory amplifiers.

This work has been realized in the context of internal CNES project A.T.F. (“Atelier Telecom du Futur”).

\section{REFERENCES}

[1] J. Sombrin, 'Critère de comparaison, d'optimisation et d'utilisation optimale des amplificateurs de puissance non linéaires", CNES report, ref. CNES DT-96-16CT/AE/TTL/HY, may 1989.

[2] C. Boulanger, A. Mallet, J. Puech, L. Lapierre, J. Sombrin, "A new criterion for power amplifier comparison and optimization", colloquium on RF and microwave amplifier, London, december 2000.

[3] D. Barataud, A. Mallet, J.C Lalaurie, L. Lapierre, J. Sombrin, T. Reveyrand, J.M Nébus, "A new measurement system for large bandwith NPR characterization of satellite transponders SSPAs", ECSC 5, topics : 'On board technologies', december 1999.

[4] Agilent Technologies, "Agilent E2507B/E2508A Noise Power Ratio measurements using the Agilent E2507B/E2508A multiformat communications signal simulator", product note, www.agilent.com/find/assist

[5] Noise/Com, "Test Set speeds NPR measurements", Microwave \& RF, pp. 126-128, january 1994.

[6] J.I. Upshur, R.K. Gupta, A.E. Williams and R.T. Kroll, "Evaluation of linearity characterization techniques for multicarrier solid-state power amplifiers", IEEE MTT-S Digest, pp.47-52, 1999.

[7] S-W. Chen, W. Panton and R. Gilmore, "Effects of nonlinear distorsion on CDMA communication systems", IEEE Transactions on mirowave theory and techniques, vol. 44, n0 12, pp. 2743-2750, december 1996.

[8] A. Silberger, "The effect of nonlinear amplifiers on orthogonal-CDMA link and measures of performance, using a simplified amplifier model", pp. 521-524, 1997.

[9] J.C. Pedro, N.B. Carvalho, "A novel nonlinear distorsion characterisation standard for $\mathrm{RF}$ and microwave communication systems", Engineering science and education journal, pp.113-119, june 2001. 